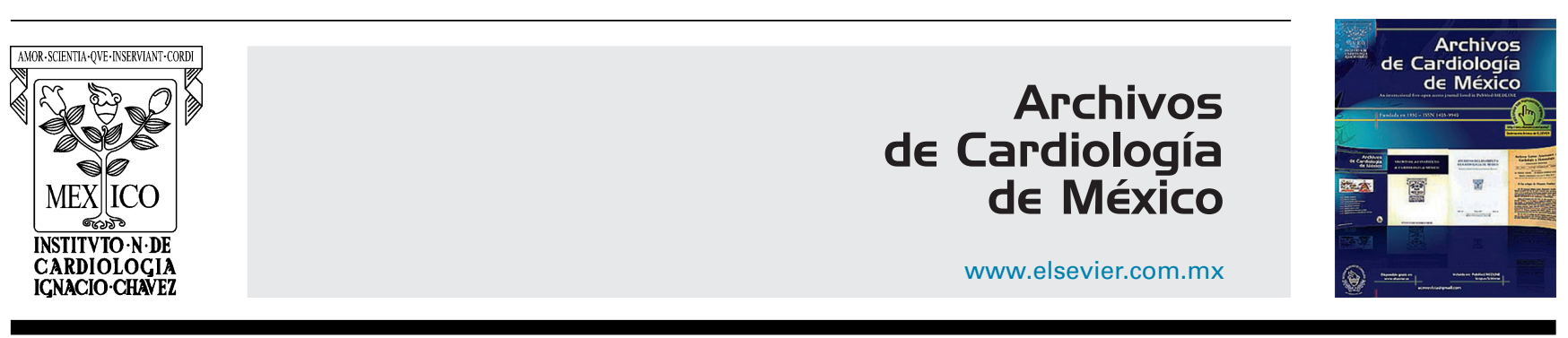

INVESTIGACIÓN CLÍNICA

\title{
Marcadores angiográficos negativos en la intervención coronaria percutánea de las oclusiones totales crónicas
}

\author{
Mohsen Mohandes*, Jordi Guarinos, Judit Rodríguez, Gil Bonet, \\ Francisco Fernández, Sergio Rojas, Cristina Moreno, Ramón de Castro, \\ Jordi Mercé y Alfredo Bardají
}

Unidad de Hemodinámica, Servicio de Cardiología, Hospital Universitario Joan XXIII, IISPV, Universitat Rovira i Virgili, Tarragona, España

Recibido el 11 de agosto de 2016; aceptado el 16 de enero de 2017

PALABRAS CLAVE
Variables
angiográficas;
Intervención
coronaria
percutánea;
Oclusión total
crónica;
España

PALABRAS CLAVE angiográficas; Intervención coronaria percutánea; crónica; España

\begin{abstract}
Resumen
Objetivos: El objetivo de este estudio es evaluar variables angiográficas predictivas negativas y la presencia de la rama lateral en la proximidad de la capa distal de la oclusión en el resultado de la intervención coronaria percutánea de las oclusiones totales crónicas.

Método: Las variables angiográficas potencialmente negativas fueron evaluadas retrospectivamente en 156 oclusiones totales crónicas sometidas a intervención coronaria percutánea. Se utilizó regresión logística binaria con una finalidad predictiva para identificar un modelo de variables que en su conjunto puedan predecir satisfactoriamente el resultado negativo de la intervención.

Resultados: Las variables asociadas de forma independiente al fracaso de procedimiento fueron la enfermedad multivaso (odds ratio = 5,12; intervalo de confianza del 95\%, 1,94-13,5; P=0.001), la presencia de muñón ambiguo (odds ratio =5,08; IC intervalo de confianza del 95\%, 2,2211,$63 ; \mathrm{P}<0.001$ ), longitud de la oclusión $\geq 20 \mathrm{~mm}$ (odds ratio $=3,7 ; \mathrm{IC}$ intervalo de confianza del $95 \%, 1,37-9,97 ; P=0.01$ ) y la localización ostial de la oclusión (odds ratio $=6,53$; intervalo de confianza del 95\%, 1,67-25,63; $\mathrm{P}=0.007)$. La rama lateral en la proximidad de la capa distal no permaneció en el modelo predictivo.

Conclusión: La enfermedad multivaso, muñón ambiguo, una longitud $\geq 20 \mathrm{~mm}$ y la localización ostial son factores independientes y predictivos de un resultado desfavorable de la angioplastia. La rama lateral en la capa distal de la oclusión no se asoció al fracaso de la intervención.

(c) 2017 Instituto Nacional de Cardiología Ignacio Chávez. Publicado por Masson Doyma México S.A. Este es un artículo Open Access bajo la licencia CC BY-NC-ND (http://creativecommons. org/licenses/by-nc-nd/4.0/).
\end{abstract}

\footnotetext{
* Autor para correspondencia. C/Dr. Mallafrè Guasch, 4; 43007; Tarragona; Hospital Universitario Joan XXIII; Unidad de Hemodinámica; Teléfono: +977295817; fax: +977295817.

Correo electrónico: mohandesmohsen@hotmail.com (M. Mohandes).
} 


\section{KEYWORDS}

Angiographic

variables;

Percutaneous

coronary

intervention;

Chronic total

occlusion;

Spain
Negative angiographic markers in percutaneous coronary intervention of chronic total occlusions

\begin{abstract}
Objective: The purpose of this study is to identify negative angiographic predictive variables and the presence of a side branch close to the distal cap of the occlusion in the chronic total occlusion percutaneous coronary intervention outcome.

Methods: Potential negative angiographic variables were retrospectively evaluated in 156 chronic total occlusions that had undergone a percutaneous coronary intervention. Binary logistic regression with predictive purpose was used to identify a model of variables which, all in all, could successfully predict a negative intervention result.

Results: Variables independently associated with the procedural failure were multivessel disease (odds ratio $=5.12 ; 95 \%$ confidence interval $(\mathrm{Cl}) ; 1.94-13.5 ; P=.001$ ), ambiguous stump presence (odds ratio $=5.08 ; 95 \% \mathrm{Cl} ; 2.22-11.63 P<.001$ ), occlusion length $\geq 20 \mathrm{~mm}$ (odds ratio $=3.7 ; 95 \% \mathrm{Cl} ; 1.37-9.97 P=.01$ ), and ostial location (odds ratio $=6.53 ; 95 \% \mathrm{Cl} ; 1.67-25.63$; $P=.007)$. Side branch at distal cap proximity did not remain in the predictive model.

Conclusions: Multivessel disease, ambiguous stump, a length $\geq 20 \mathrm{~mm}$, and an ostial location of a chronic total occlusion are independent predictive factors of an unfavourable angioplasty result. A side branch at occlusion distal cap was not associated with the procedural failure.

(c) 2017 Instituto Nacional de Cardiología Ignacio Chávez. Published by Masson Doyma México S.A. This is an open access article under the CC BY-NC-ND license (http://creativecommons. org/licenses/by-nc-nd/4.0/).
\end{abstract}

\section{Introducción}

La oclusión total crónica (OTC) de una arteria coronaria se define como la obstrucción total de la arteria coronaria con flujo TIMI 0 y con más de 3 meses de duración ${ }^{1}$. En un registro reciente la prevalencia de OTC en pacientes con enfermedad coronaria, definida como la presencia de lesión angiográfica de más $50 \%$ en al menos una arteria coronaria, fue de $18.2 \%$ mientras que la misma prevalencia en el grupo de pacientes sometidos a cirugía de revascularización coronaria previa se estimó de $54 \%$ y la prevalencia de OTC en pacientes sometidos a angioplastia primaria fue de $10 \%^{2}$. La presencia de una OTC se ha considerado como una de las causas más comunes por las que se remite al paciente a cirugía de bypass coronario $^{3}$. A pesar de múltiples avances en los últimos años en términos de aparición de nuevas técnicas y materiales específicos, la intervención coronaria percutánea (ICP) de las OTC supone uno de los terrenos más desafiantes en cardiología intervencionista y las tasas de éxito de ICP en este tipo de procedimientos son considerablemente más bajas que las de las lesiones no oclusivas ${ }^{4}$ habiéndose situado en 70 y $98 \%$ respectivamente ${ }^{5}$.

Por otra parte estudios no aleatorizados muestran que la recanalización exitosa de una OTC, en era de stents liberadores de droga, se asocia a largo plazo, a la mejora de la supervivencia comparada con el grupo de pacientes en los que la ICP sobre OTC es fallida ${ }^{6}$.

Históricamente se han identificado algunos marcadores angiográficos como potenciales predictores negativos en la ICP de las OTC como la ausencia de flujo anterógrado (oclusión funcional), la existencia de un muñón ambiguo, la presencia de calcificación, tortuosidad importante del vaso y/o del segmento de oclusión, la localización ostial de la oclusión, presencia de una rama lateral en el comienzo del segmento de oclusión, diámetro arterial menor de $2.5 \mathrm{~mm}$, falta de visibilidad del campo distal, enfermedad multivaso, longitud del segmento de oclusión y la presencia de colaterales puente ${ }^{7-12}$.

La influencia de algunas de estas variables se ha reforzado en diferentes estudios a lo largo de los últimos años mientras que otras como la presencia de colaterales puente han perdido fuerza en parte gracias a avances en materiales específicos, adelantos en técnicas de procedimiento e incremento en experiencia de los operadores ${ }^{13}$.

El objetivo de este estudio fue identificar los marcadores angiográficos predictivos del fracaso de la ICP en nuestro centro y evaluar la eventual influencia de la variable proximidad de la capa distal de la OTC con una rama lateral y tratar de construir un modelo que pueda predecir satisfactoriamente el riesgo de fracaso de la intervención.

\section{Métodos}

Se trata de un estudio retrospectivo de la serie de ICP sobre OTC realizadas en nuestro centro entre mayo de 2007 y agosto de 2013 en los que se analizaron las variables angiográficas potencialmente negativas para el resultado de la ICP. La inclusión de OTC se basó en la definición del documento de consenso del grupo EuroCTO ${ }^{1}$. Se analizaron las variables cuya influencia negativa en cuanto al resultado del procedimiento tenían una base teórica basada en estudios anteriores. Estas variables fueron la ausencia de un muñón bien definido, es decir, muñón ambiguo, la enfermedad multivaso, la proximidad de la capa proximal de la OTC con una rama lateral, la presencia de calcificación visible en la angiografía en el segmento de la oclusión, la longitud de la oclusión, la visibilidad del campo distal de la arteria coronaria, localización ostial de la oclusión, el 
diámetro arterial menor de $2.5 \mathrm{~mm}$ y la tortuosidad del vaso y/o segmento de OTC. Se evaluó además la variable localización de la capa distal del segmento de la OTC en la proximidad de una rama lateral por tener un sentido lógico ya que en ocasiones, una vez cruzada buena parte del segmento de oclusión, la entrada definitiva de la guía en luz verdadera distal puede verse dificultada por presencia de una rama lateral. La evaluación de esta variable fue limitada a aquellos casos en los que la rama lateral fuera $\geq 1.5 \mathrm{~mm}$ con un ángulo respecto al campo distal de la arteria principal de menos de 90 grados y que este vaso a su vez no fuera potencialmente candidato para la angioplastia. Dos observadores independientes analizaron y determinaron valores de las correspondientes variables y en casos de difícil interpretación se trató o bien de consensuar o bien intervino un tercer observador para asignar el valor definitivo. Los observadores no fueron ciegos de forma sistemática respecto al resultado del procedimiento. Una vez identificada una OTC la decisión sobre la ICP se basó en la clínica del paciente y en la evidencia de viabilidad y/o isquemia en el territorio correspondiente a la arteria coronaria. Los pacientes que no mostraban signos de necrosis electrocardiográfica y no presentaban aquinesia de este territorio en el examen ecocardiográfico y presentaban síntomas eran elegidos para la ICP. En presencia de necrosis electrocardiográfica y/o aquinesia en el ecocardiograma se procedía a realizar pruebas de detección de isquemia con SPECT miocárdico o demostración de viabilidad mediante RMN cardíaca. Los procedimientos se llevaban a cabo principalmente de forma diferida y tras diseñar la estrategia en cada caso y discutir en equipo las dificultades de cada intervención. El éxito del procedimiento se definió cuando se conseguía restaurar el flujo $\geq$ TIMI II con una lesión residual menor de $30 \%$.

\section{Procedimiento}

Todos los pacientes estaban bajo tratamiento con doble antiagregación con ácido acetilsalicílico y clopidogrel. Si se identificaba circulación colateral heterocoronaria, aunque fuera escasa, se usaba inyección contralateral simultánea con catéter guía para visualizar mejor el campo distal de la OTC. La arteria coronaria ocluida se sondaba preferiblemente con catéteres guías 7-8 French $(\mathrm{Fr})$ y principalmente por el abordaje femoral salvo casos donde se preveía posible el uso de catéteres $7 \mathrm{Fr}$ por el acceso radial. Se hacía determinación de ACT durante el procedimiento cada $30 \mathrm{~min}$ para mantener niveles entre 250-300 segundos en casos de acceso anterógrado y cifras por encima de 300 en abordaje retrógrado. Se usaban guías floppy apoyadas sobre microcatéteres específicos para llegar a la capa proximal del segmento de oclusión y a continuación se intercambiaba con guías diseñadas para cruzar el segmento de oclusión de una manera escalonada de menor a mayor poder de penetración siendo la guía de primera elección para acceso anterógrado la Fielder XT (Asahi Intecc; Aichi, Japón). Una vez cruzado el segmento de oclusión se hacía comprobación de la posición de la guía en luz verdadera mediante inyección en dos proyecciones ortogonales diferentes. Una vez asegurada la posición de la guía en luz verdadera se procedía a atravesar el segmento de oclusión con microcatéter e inmediatamente se intercambia la guía con una convencional para continuar con la angioplastia. En todos los casos, salvo contraindicación, se implantaba stent farmacoactivo.

\section{Análisis estadístico}

Las distintas variables angiográficas (tabla 1) se introdujeron en un modelo multivariante con regresión logística binaria con una finalidad predictiva buscando el conjunto de variables que pudieran predecir satisfactoriamente el fracaso del procedimiento. La variable longitud del segmento de oclusión se introdujo como continua y posteriormente se categorizó sobre el nivel de corte $20 \mathrm{~mm}$ para su posterior introducción en el modelo. Las demás variables angiográficas se introdujeron en la base de datos de forma categórica binaria. Para elegir el modelo predictivo se utilizó el método de selección de variables por pasos utilizando la estrategia de inclusión (FSTEP) secuencial. A partir del modelo final elegido se estimó la curva de ROC, su intervalo de confianza (IC) y su nivel de significación para determinar la validez predictiva del modelo. La fiabilidad del modelo final se evaluó segmentando la muestra en una proporción de 1/3 para muestra de validación y de $2 / 3$ para muestra de derivación restando la diferencia de área bajo curva (AUC) de ambas muestras para calcular de esta manera la pérdida de predicción. A partir del modelo final se construyó una tabla de índices pronósticos consistente en el cálculo de riesgo de fracaso del procedimiento basado en combinación de diferentes valores de las variables del modelo en sujetos ficticios. Asimismo se calculó el riesgo relativo del fracaso tomando como referencia el patrón de valores de menor riesgo $^{14}$. La comparación de variables categóricas se llevó a cabo con el estadístico $\mathrm{X}^{2}$ de Pearson y test exacto de Fisher cuando fuera necesario y las variables continuas se compararon con t de student si la distribución de la variable era normal y con prueba no paramétrica de U de Mann-Whitney si la distribución de la variable vulneraba los principios de la normalidad. Las variables continuas se expresaron con media y desviación estándar y las variables discretas con número y porcentajes. Se consideró significación estadística niveles de $p<0.05$. Se utilizó el paquete estadístico SPSS 19 para analizar los datos y para elaborar la sintaxis de las operaciones estadísticas.

\section{Resultados}

Se llevaron a cabo 156 ICP sobre OTC en 131 pacientes entre mayo de 2007 y agosto de 2013. La comparación de las características basales entre el grupo de éxito y fracaso de ICP se muestra en la (tabla 2) no existiendo diferencias significativas entre ambos grupos. La media de edad de los pacientes fue $63 \pm 10.3$ años y 138 (88.5\%) de ellos eran varones. No se observaron diferencias significativas en las características de ambos grupos. En 62(39.7\%) de los casos se utilizó doble inyección femoral-radial, en $32(20.5 \%)$ doble inyección femoral-femoral, en $1(0.6 \%)$ doble inyección femoral-cubital, en $1(0.6 \%)$ doble inyección radial-radial, en $35(22.4 \%)$ se utilizó solo abordaje femoral y finalmente en 25 (16\%) solo el acceso radial. En 135 (86.5\%) el procedimiento se llevó a cabo por el abordaje anterógrado y en 21 (13.5\%) se utilizó el acceso retrógrado o bien 
Tabla 1 Comparación de características angiográficas entre grupos de éxito y fracaso de ICP

\begin{tabular}{lccc}
\hline Variable & Éxito $(\mathrm{N}=112)$ & Fracaso $(\mathrm{N}=44)$ & $\mathrm{P}$ \\
\hline Multivaso & $60(53.6 \%)$ & $34(77.3 \%)$ & 0.006 \\
Calcificación & $43(38.4 \%)$ & $15(34.1 \%)$ & 0.62 \\
Muñón ambiguo & $29(25.9 \%)$ & $27(61.4 \%)$ & 0.001 \\
Rama lateral proximal & $52(46.4 \%)$ & $33(75 \%)$ & 0.001 \\
Rama lateral distal & $22(19.6 \%)$ & $9(20.5 \%)$ & 0.9 \\
Tortuosidad & $30(26.8 \%)$ & $16(36.4 \%)$ & 0.24 \\
Localización ostial & $5(4.5 \%)$ & $7(15.9 \%)$ & 0.039 \\
Diámetro $<2,5 \mathrm{~mm}$ & $39(34.8 \%)$ & $17(38.6 \%)$ & 0.66 \\
Visibilidad subóptima & $17(15.2 \%)$ & $14(18.2 \%)$ & 0.65 \\
Longitud OTC $\geq 20 \mathrm{~mm}$ & $4(12.5 \%)$ & & 0.005 \\
\hline
\end{tabular}

OTC: oclusión total crónica.

Tabla 2 Comparación de características basales entre grupos de éxito y fracaso de ICP

\begin{tabular}{|c|c|c|c|c|}
\hline Variable & Total (156) & Éxito $(\mathrm{N}=112)$ & Fracaso $(\mathrm{N}=44)$ & $P$ \\
\hline Edad (media $\pm S D)$ & $63 \pm 10.3$ & $62 \pm 10.72$ & $64.5 \pm 9.7$ & 0.2 \\
\hline $\operatorname{Sexo}(H)$ & $138(88.5 \%)$ & 97 (86.6\%) & $41(93.2 \%)$ & 0.25 \\
\hline HTA & $116(74.4 \%)$ & 85 (75.9\%) & $31(70.5 \%)$ & 0.48 \\
\hline Dislipemia & $115(73.7 \%)$ & 82 (73.2\%) & $33(75 \%)$ & 0.82 \\
\hline Diabetes & 70 (44.9\%) & $47(42 \%)$ & $23(52.3 \%)$ & 0.24 \\
\hline Tabaquismo & $100(64.1 \%)$ & $71(63.4 \%)$ & $29(65.9 \%)$ & 0.77 \\
\hline Diagnóstico & & & & 0.76 \\
\hline Angina estable & $119(76.3 \%)$ & 85 (75.9\%) & $34(77.3 \%)$ & \\
\hline SCASEST & 32 (20.5\%) & $24(21.4 \%)$ & $8(18.2 \%)$ & \\
\hline ICC & $5(3.2 \%)$ & $3(2.7 \%)$ & $2(4.5 \%)$ & \\
\hline FE & & & & 0.59 \\
\hline$>50 \%$ & 108 (69.2\%) & $76(71 \%)$ & $32(72.7 \%)$ & \\
\hline $40-50 \%$ & $19(12.2 \%)$ & $13(12.1 \%)$ & $6(13.6 \%)$ & \\
\hline $30-40 \%$ & $17(10.9 \%)$ & $14(13.1 \%)$ & $3(6.8 \%)$ & \\
\hline$<30 \%$ & $7(4.5 \%)$ & $4(3.7 \%)$ & $3(6.8 \%)$ & \\
\hline Arteria tratada & & & & 0.93 \\
\hline DA & $54(34.6 \%)$ & $40(35.7 \%)$ & $14(31.8 \%)$ & \\
\hline DG & $1(0.6 \%)$ & $1(0.9 \%)$ & $0(0 \%)$ & \\
\hline$C X$ & $37(23.7 \%)$ & $26(23.2 \%)$ & $11(25 \%)$ & \\
\hline$C D$ & $64(41 \%)$ & 45 (40.2\%) & $19(43.2 \%)$ & \\
\hline
\end{tabular}

CD: coronaria derecha. CX: circunfleja; DA: descendente anterior; DG: diagonal; DSVI: disfunción sistólica del ventrículo izquierdo; FE: fracción de eyección; HTA: hipertensión arterial; ICC: insuficiencia cardíaca congestiva; ICP: intervención coronaria percutánea.

como técnica complementaria al anterógrado o bien como primera elección. En 112 (71.8\%) de los casos el procedimiento fue exitoso y en 44 (28.2\%) fallido. La tasa de éxito total por paciente y arteria tratada fue de $80 \%$. La enfermedad multivaso, la existencia de muñón ambiguo, la presencia de rama lateral en capa proximal, la localización ostial y la longitud de oclusión $\geq 20 \mathrm{~mm}$ fueron estadísticamente más frecuentes en el grupo de ICP fallida (tabla 1). Las variables angiográficas independientes predictivas del fracaso del procedimiento en el modelo multivariante fueron la enfermedad multivaso, la presencia de muñón ambiguo, longitud de la oclusión $\geq 20 \mathrm{~mm}$ y la localización ostial de la oclusión (tabla 3). La variable presencia de la rama lateral en proximidad de capa distal no permaneció en el modelo predictivo no siendo una variable predictiva negativa para ICP. La curva de ROC mostró un área de 0.8 con IC 95\%, 0.721-0.873
Tabla 3 Variables predictivas negativas en el modelo multivariante con regresión logística binaria

\begin{tabular}{llrc}
\hline Variable & OR & \multicolumn{1}{c}{ IC de 95\% } & \multicolumn{1}{c}{$\mathrm{P}$} \\
\hline Enfermedad multivaso & 5.12 & $1.94-13.5$ & 0.001 \\
Muñón ambiguo & 5.08 & $2.22-11.63$ & $<0.001$ \\
Longitud $\geq 20 \mathrm{~mm}$ & 3.7 & $1.37-9.97$ & 0.01 \\
Localización ostial & 6.53 & $1.67-25.63$ & 0.007 \\
\hline
\end{tabular}

IC: intervalo de confianza; OR: odds ratio.

(fig. 1). El modelo presentó una pérdida de predicción (diferencia de AUC entre muestra de validación y muestra de derivación) de $5 \%$. El cálculo de índices pronósticos para sujetos ficticios mostró el patrón de menor riesgo para el fracaso de ICP sobre la OTC aquel que no mostraba ningún 


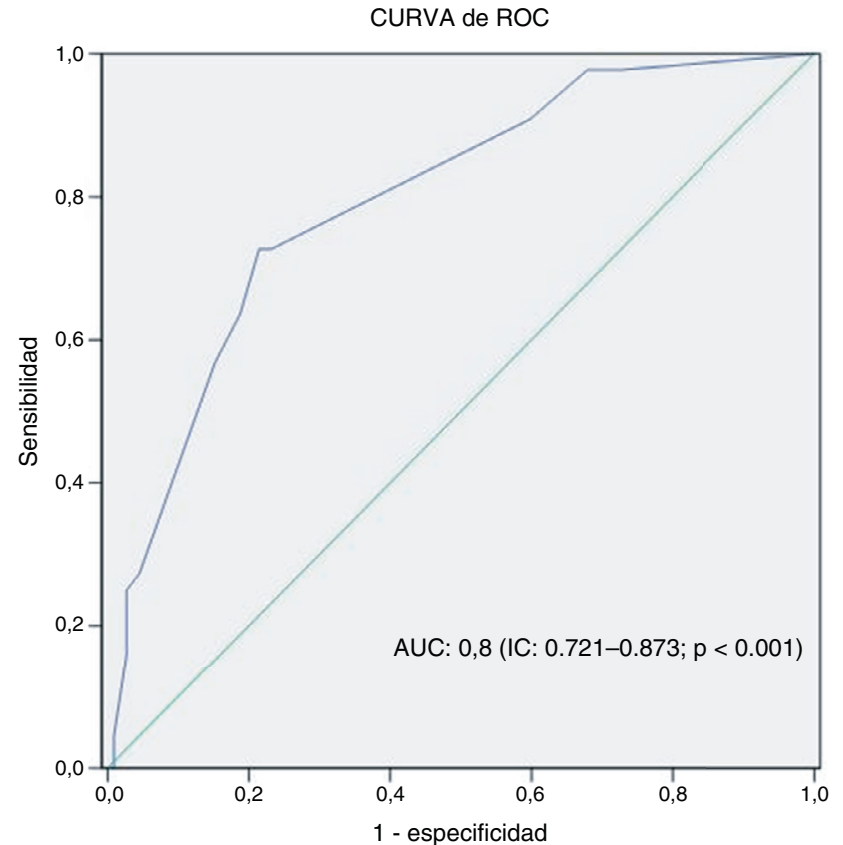

Figura 1 Curva de ROC con su intervalo de confianza y su nivel de significación.

AUC: área bajo la curva; IC: intervalo de confianza.

valor desfavorable de las 4 variables finalistas del modelo siendo el riesgo de este patrón de 0.0392 . El patrón más desfavorable y propenso al fracaso de la intervención fue la combinación de valores desfavorables en las 4 variables con un riesgo relativo de fracaso de 24.5 veces mayor respecto al patrón de referencia, es decir el de menor riesgo (tabla 4).

\section{Discusión}

Los resultados de este estudio sugieren que el conjunto de las variables angiográficas que predicen razonablemente el fracaso de la ICP sobre una OTC es la enfermedad multivaso, la presencia de un muñón ambiguo, una longitud del segmento de oclusión $\geq 20 \mathrm{~mm}$ y la localización ostial de la oclusión. Este modelo presenta una capacidad predictiva razonable como muestra la curva de ROC (0.8). La variable rama lateral en la proximidad de la capa distal del segmento de la oclusión no fue un factor independiente predictivo del fracaso de la angioplastia.

La identificación de marcadores angiográficos potencialmente desfavorables para el resultado de la ICP en OTC ha sido objeto de muchos estudios. La ICP sobre OTC tiene sus peculiaridades técnicas y precisa un entrenamiento especial y es importante el diseño de estrategia previo a la intervención y conocer el grado de dificultad que supone cada caso. En nuestro estudio nos centramos en analizar la influencia de variables angiográficas como potenciales factores predictivos negativos cuya influencia había sido demostrada en estudios anteriores y se basaba en un sentido lógico en cuanto a la dificultad que suponían estos marcadores a la hora de recanalizar la oclusión. La variable longitud del segmento de oclusión con el punto de corte $20 \mathrm{~mm}$ ha sido
Tabla 4 Riesgo relativo (RR) de diferentes patrones según la combinación de valores (tener $=1$ o no tener $=0$ un valor desfavorable) de variables del modelo en sujetos teóricos. El RR se ha estimado respecto al patrón de referencia (patrón de menor riesgo con una probabilidad de fracaso de ICP: 0.0392 en primera fila)

\begin{tabular}{lllll}
\hline Multivaso & Longitud OTC & Muñón & Ostial & RR \\
\hline 0 & 0 & 0 & 0 & 1 \\
0 & 1 & 0 & 0 & 3.34 \\
0 & 0 & 1 & 0 & 4.38 \\
1 & 0 & 0 & 0 & 4.40 \\
0 & 0 & 0 & 1 & 5.37 \\
0 & 1 & 1 & 0 & 11.07 \\
1 & 1 & 0 & 0 & 11.11 \\
0 & 1 & 0 & 1 & 12.66 \\
1 & 0 & 1 & 0 & 13.13 \\
0 & 0 & 1 & 1 & 14.67 \\
1 & 0 & 0 & 1 & 14.71 \\
1 & 1 & 1 & 0 & 20.31 \\
0 & 1 & 1 & 1 & 21.25 \\
1 & 1 & 0 & 1 & 21.27 \\
1 & 0 & 1 & 1 & 22.27 \\
1 & 1 & 1 & 1 & 24.53 \\
\hline 0
\end{tabular}

OTC: oclusión total crónica; RR: riesgo relativo.

La negrita indica el patrón de referencia (el de menor riesgo de fracaso; cuando las 4 variables: multivaso, longitud OTC, muñón y ostial tienen un valor 0 ). Los siguientes riesgos relativos de la columna se han estimado respecto a este patrón de referencia.

estudiada demostrando su impacto negativo en el resultado de la ICP de OTC en estudios previos ${ }^{9,15}$ aunque el nivel de corte $15 \mathrm{~mm}$ también ha sido evaluado y su impacto negativo demostrado ${ }^{16}$. En nuestro análisis elegimos el nivel de corte $20 \mathrm{~mm}$ por haber sido constatada su influencia de forma repetida en estudios más recientes. Tomasello et al. ${ }^{12}$, analizaron 328 OTC y mostraron que junto con longitud $>20 \mathrm{~mm}$, las variables calcificación severa y el diámetro del vaso $<2.5 \mathrm{~mm}$ eran variables angiográficas asociadas al fracaso del procedimiento. Estudios recientes también corroboran el impacto negativo de la calcificación excesiva, tortuosidad importante y ausencia de muñón como predictores independientes del fracaso de la angioplastia ${ }^{8}$. En el presente artículo hemos utilizado el término muñón ambiguo cuando el operador no puede identificar de forma clara la desembocadura del segmento de la oclusión. Este hallazgo equivale al término ausencia del muñón afilado en otras publicaciones.

Existe un hecho destacable entre los diferentes estudios, incluido el nuestro, que no todos identifican las mismas variables angiográficas como predictoras negativas de la ICP sobre OTC. Este hecho puede deberse en parte a la diferencia en el diseño, el tamaño de la muestra, la inclusión en algunos estudios de variables clínicas y el distinto grado de experiencia en el abordaje de OTC de diferentes centros. Este último factor, se sabe, que es muy determinante en el resultado final de este tipo de procedimientos. En este sentido los resultados de nuestro estudio representan a un único centro con experiencia progresiva en el manejo de CTO y acumulada prácticamente sobre un solo operador. 
Aunque en nuestro estudio se han valorado las variables angiográficas, algunas de las cuales con influencia negativa muy demostrada previamente sobre el resultado de la ICP, la variable presencia de un ramo lateral en la proximidad de la capa distal no había sido evaluada con anterioridad y su inclusión en el análisis desde nuestro punto de vista tiene un sentido lógico. Este hecho tiene una relevancia especialmente en algunos procedimientos con oclusiones relativamente largas y tortuosas ya que una vez cruzado el cuerpo principal de la oclusión, la presencia de una rama lateral distal puede dificultar el redirigir la guía hacia el campo distal de la arteria y podría eventualmente influir en el resultado final de la intervención. Por tanto, creemos que era necesario analizar la posible influencia negativa de esta variable que los resultados de nuestro estudio no la sugieren.

Por otra parte, la ICP de una OTC difiere en gran parte de una angioplastia convencional y precisa mayor consumo de tiempo, uso de materiales específicos, entrenamiento especial y mayor nivel de exposición a la irradiación ${ }^{17-19}$. Estos factores han influido en parte a que el intervencionismo sobre OTC esté menos extendido que otras angioplastias más convencionales en laboratorios de hemodinámica. En este sentido es muy importante, además de entrenamiento específico, una correcta selección de los casos y el incremento escalonado de la complejidad de las intervenciones. Nuestro estudio, aunque se limita a un único centro, puede reflejar el escenario de una sala de hemodinámica donde se implanta el programa de OTC con un nivel de experiencia progresivo y en este sentido la identificación de variables angiográficas desfavorables y la disponibilidad de un índice de riesgo que nos oriente hacia el resultado de la intervención puede ayudar al operador en la selección de los casos menos desfavorables en las primeras etapas de su experiencia con el fin de incrementar progresivamente el nivel de complejidad tal como recomienda EuroCTO club $^{20}$. La adquisición progresiva de experiencia y compartir casos complejos con otros operadores es altamente recomendable en este tipo de intervenciones.

\section{Limitaciones}

Nuestro estudio tiene varias limitaciones. En primer lugar es un estudio retrospectivo y unicéntrico con una muestra relativamente pequeña y sus resultados no son extrapolables a cualquier otro centro, a tener en cuenta que el resultado final de este tipo de intervenciones es muy dependiente del nivel de experiencia del operador. En este sentido se sabe que el abordaje retrógrado unido a técnicas complejas como CART o CART inverso puede superar algunas dificultades en casos con anatomía desfavorable ${ }^{21,22}$. Dado que la incorporación de técnicas complejas en nuestro centro se ha materializado en etapas más avanzadas de la implantación del programa de OTC los resultados obtenidos pueden estar parcialmente sesgados. Otra limitación de nuestro estudio es el hecho de que los observadores no estaban ciegos de forma sistemática respecto al resultado del procedimiento a la hora de medir las variables.

\section{Conclusiones}

En nuestro estudio la enfermedad multivaso, muñón ambiguo, una longitud $\geq 20 \mathrm{~mm}$ y la localización ostial de la oclusión son factores independientes y predictivos de un resultado desfavorable de la angioplastia sobre una OTC. Este modelo puede predecir el resultado negativo de ICP sobre OTC con una validez predictiva y una fiabilidad razonables. La presencia de rama lateral en la capa distal de la oclusión no se asoció al fracaso de la intervención. La elaboración de un índice pronóstico para estimar el riesgo de un resultado de ICP desfavorable, según la combinación de diferentes patrones angiográficos, puede orientar al operador en abordar este tipo lesiones de manera escalonada y de acuerdo al nivel de complejidad. Estudios multicéntricos y prospectivos con mayor número de pacientes son necesarios para sacar conclusiones definitivas.

\section{Responsabilidades éticas}

Protección de personas y animales. Los autores declaran que los procedimientos seguidos se conformaron a las normas éticas del comité de experimentación humana responsable y de acuerdo con la Asociación Médica Mundial y la Declaración de Helsinki.

Confidencialidad de los datos. Los autores declaran que han seguido los protocolos de su centro de trabajo sobre la publicación de datos de pacientes.

Derecho a la privacidad y consentimiento informado. Los autores declaran que en este artículo no aparecen datos de pacientes.

\section{Financiación}

No se recibió patrocinio de ningún tipo para llevar a cabo este artículo.

\section{Conflicto de intereses}

Los autores declaran no tener ningún conflicto de intereses.

\section{Agradecimientos}

Los autores agradecen profundamente la aportación valiosa de todos los miembros de la Unidad de Hemodinámica del Hospital Joan XXIII en la materialización de los procedimientos.

\section{Bibliografía}

1. Sianos G, Werner GS, Galassi AR, et al. Recanalisation of chronic total coronary occlusions: 2012 consensus document from the EuroCTO club. Eurolntervention. 2012;8:139-45.

2. Fefer P, Knudtson ML, Cheema AN, et al. Current perspectives on coronary chronic total occlusions: The canadian multicenter chronic total occlusions registry. J Am Coll Cardiol. 2012;59:991-7. 
3. Srinivas VS, Brooks MM, Detre KM, et al. Contemporary percutaneous coronary intervention versus balloon angioplasty for multivessel coronary artery disease: A comparison of the national heart, lung and blood institute dynamic registry and the bypass angioplasty revascularization investigation (BARI) study. Circulation. 2002;106:1627-33.

4. Stone GW, Kandzari DE, Mehran R, et al. Percutaneous recanalization of chronically occluded coronary arteries: A consensus document: Part I. Circulation. 2005;112:2364-72.

5. Prasad A, Rihal CS, Lennon RJ, et al. Trends in outcomes after percutaneous coronary intervention for chronic total occlusions: A 25-year experience from the mayo clinic. J Am Coll Cardiol. 2007;49:1611-8.

6. Jones DA, Weerackody R, Rathod K, et al. Successful recanalization of chronic total occlusions is associated with improved long-term survival. JACC Cardiovasc Interv. 2012;5:380-8.

7. Ivanhoe RJ, Weintraub WS, Douglas JS Jr, et al. Percutaneous transluminal coronary angioplasty of chronic total occlusions: primary success, restenosis, and long-term clinical follow-up. Circulation. 1992;85:106-15.

8. Li CG, Liu XB, Ge L, et al. Determinants of success percutaneous coronary intervention in patients with chronic total coronary artery occlusion. Zhonghua Xin Xue Guan Bing Za Zhi. 2011;39:30-4.

9. Noguchi T, Miyazaki MDS, Morii I, et al. Percutaneous transluminal coronary angioplasty of chronic total occlusions. Determinants of primary success and long-term clinical outcome. Catheter Cardiovasc Interv. 2000;49:258-64.

10. Suzuki T, Hosokawa H, Yokoya K, et al. Time-dependent morphologic characteristics in angiographic chronic total coronary occlusions. Am J Cardiol. 2001;88:A5-6.

11. Stone GW, Colombo A, Teirstein PS, et al. Percutaneous recanalization of chronically occluded coronary arteries: Procedural techniques, devices, and results. Catheter Cardiovasc Interv. 2005; 66:217-36.

12. Tomasello SD, Costanzo L, Campisano MB, et al. Does occlusion duration influence procedural and clinical outcome of patients who underwent percutaneous coronary intervention for chronic total occlusion. J Interv Cardiol. 2011;24:223-31.
13. Kinoshita I, Katoh O, Nariyama J, et al. Coronary angioplasty of chronic total occlusions with bridging collateral vessels: Immediate and follow-up outcome from a large single-center experience. J Am Coll Cardiol. 1995;26:409-15.

14. Doménech JM, Navarro JB. Regresión logística binaria, multinomial, de poisson y binomial negativa. 6. $\underline{a}$ ed. Barcelona: Signo; 2011.

15. Morino Y, Abe M, Morimoto T, et al. Predicting successful guidewire crossing through chronic total occlusion of native coronary lesions within 30 minutes: The J-CTO (multicenter CTO registry in japan) score as a difficulty grading and time assessment tool. JACC Cardiovasc Interv. 2011;4:213-21.

16. Olivari Z, Rubartelli P, Piscione F, et al. Immediate results and one-year clinical outcome after percutaneous coronary interventions in chronic total occlusions: Data from a multicenter, prospective, observational study (TOAST-GISE). J Am Coll Cardiol. 2003;41:1672-8.

17. Grantham JA, Marso SP, Spertus J, et al. Chronic total occlusion angioplasty in the united states. JACC Cardiovasc Interv. 2009;2:479-86.

18. Suzuki S, Furui S, Isshiki T, et al. Factors affecting the patient's skin dose during percutaneous coronary intervention for chronic total occlusion. Circ J. 2007;71:229-33.

19. Suzuki S, Furui S, Isshiki T, et al. Patients' skin dose during percutaneous coronary intervention for chronic total occlusion. Catheter Cardiovasc Interv. 2008;71:160-4.

20. Di Mario C, Werner GS, Sianos G, et al. European perspective in the recanalisation of chronic total occlusions (CTO): Consensus document from the EuroCTO club. Eurolntervention. 2007;3:30-43.

21. Surmely JF, Tsuchikane E, Katoh O, et al. New concept for СTO recanalization using controlled antegrade and retrograde subintimal tracking: The CART technique. J Invasive Cardiol. 2006;18:334-8.

22. Yamane M. Current percutaneous recanalization of coronary chronic total occlusion. Rev Esp Cardiol (Engl Ed). 2012;65:265-77. 